\title{
The influence of monomer shape on aggregate morphologies
}

\author{
J. D. Perry, E. Gostomski, L. S. Matthews, and T. W. Hyde
}

Center for Astrophysics, Space Physics, and Engineering Research, Baylor University, One Bear Place 97316 Waco, TX 76798, USA

e-mail: jonathan_perry@baylor.edu

Received 23 August 2011 / Accepted 15 December 2011

\begin{abstract}
Context. The coagulation of dust particles is the initial step in planetary formation, with the precursors to planetesimals believed to form via the collisions of micron and submicron sized dust particles in the disk surrounding a newly formed protostar. One of the usual assumptions in numerical models of aggregation is that of spherical monomers. However, the polarization of light in the interstellar medium (ISM) indicates that dust particles may not necessarily be spherical.

Aims. This study investigates the influence of monomer shape (ellipsoidal vs. spherical) on the morphology of aggregates. The ellipsoidal grains used are prolate with an axis ratio of 3:1:1, which current evidence suggests as a possible shape for instellar dust grains. Methods. Populations of aggregates are built from ellipsoidal monomers and spherical monomers using both particle-cluster aggregation (PCA) and cluster-cluster aggregation (CCA) regimes incorporating the rotation of particles. The morphology of the resulting aggregates is compared using the maximum radius, porosity, fractal dimension, compactness factor and friction time. The last two factors indicate how the dynamics of a population of dust may be altered depending on monomer shape.

Results. The results of this study indicate that monomer shape plays an important role in determining the final morphology of aggregates. Comparing ellipsoid grains with 3:1:1 axis ratio to spheres, the greatest difference is seen in compactness factors: $(\sim 18 \%)$ for the PCA regime, reaching a maximum of $(\sim 80 \%)$ for the CCA regime. The influence on porosity is also appreciable, $(\sim 8 \%)$ and $(\sim 15 \%)$ for PCA and CCA regimes respectively. The resulting differences for the friction times depend on the collision regime employed, yet show a marked difference for the different monomer shapes, $(\sim 12 \%)$ for PCA and ( $>50 \%)$ for CCA at large sizes. It is concluded that the effect of monomer shape in the hit-and-stick aggregation model may produce appreciable structural variation in the final aggregate. Present models of fluffy aggregates made up of only spherical monomers therefore may not be the best representation for grains in some astrophysical environments.
\end{abstract}

Key words. accretion, accretion disks - protoplanetary disks

\section{Introduction}

The coagulation of dust particles is an essential step in planetary formation. Precursors to planetesimals are believed to form via the collisions of micron and submicron sized dust particles in the disk surrounding a protostar. Understanding the microphysics that governs the formation and evolution of those aggregate structures is a key component to modeling the initial stages of planetary formation, atmospheric physics and processes in other coagulation environments Blum \& Wurm (2008), Skorov et al. (2008).

Several numerical models investigating the dynamics and properties of dust aggregates have recently been published Dominik \& Nubold (2002), Ormel et al. (2006), Matthews \& Hyde (2004, 2008), Perry et al. (2010). In these models, individual dust particles collide to create aggregate structures which are fractal or fluffy in nature Blum et al. (2000); Dominik et al. (2007a). The resulting structure or morphology of these aggregates are then analyzed using statistically significant populations (many thousands of particles) to determine how various properties of the resulting particles differ. One similarity between most of these models is their assumption of spherical monomers, though there is evidence via the polarization of sunlight from cometary dust suggesting the presence of dust particles with ellipsoidal shapes and axis ratios of 3:1:1 Hage \& Greenberg (1990). Assuming this data to be true, insight into the aggregation process can be gained by studying the coagulation of ellipsoids, and comparing the resulting structures to those produced using spherical monomers.

One recent study compared the aggregation of ellipsoidal and spherical monomers, using particle-cluster and clustercluster aggregation methods (PCA and CCA respectively). This study concluded that the variation in morphology produced from differing monomer shapes was too small to influence the overall structure and porosity of the resultant aggregate Bertini et al. (2009). Three different coagulation methods were employed to come to this conclusion: in the first population, PCA1, an incoming monomer had a trajectory directed towards the center of mass (COM) of the target particle; the second population, CCA, started with a small particle cluster of size $N=2,3$ or 7, replicated the cluster, rotated it to a new orientation and then collided it with a target, also using a trajectory towards the COM. The final population, PCA2, grew highly linear structures by using incoming trajectories aimed at the closest particle in the target aggregate.

The morphologies of the resulting aggregates were analyzed using porosity, fractal dimension, and friction time parameterized by the collisional cross section of the particle. It was found that the porosity of aggregates consisting of spherical or ellipsoidal monomers varied by a small amount $(\sim 5 \%)$ between populations, which is even less than the differences seen among aggregates using polydisperse populations for the initial monomers. The variance found for fractal dimension was negligible, being $<2 \%$, and shape was concluded to not have an influence on this parameter. Finally, the friction time, depending on 
the inverse of the cross-section per unit mass, showed a difference of $\sim 15 \%$ for the two aggregate types formed from CCA collisions, implying a stronger coupling to the gas for aggregates built using ellipsoidal monomers, even though this trend was concluded to be independent of the shape of the monomers. Overall the authors determined that shape was not an important factor in the aggregation process.

This study, however, did not incorporate several physical parameters necessary to realistically model aggregation in an astrophysical environment. This paper reexamines the question of the impact of monomer shape on aggregation by incorporating two new parameters, including off-center collisions and rotations of particles induced during collisions. It will be shown that although the results referenced are correct within the limitations of their assumptions, the inclusion of the these parameters can have a large impact on aggregate structure. As such, their inclusion is necessary in order to accurately model aggregation.

\section{Methods}

In this study, aggregates are initially created by numerically simulating pair-wise interactions of particles. The computer model employed has previously been used to examine the coagulation of charged particles, as well as coagulation considering the influence of magnetic materials Matthews \& Hyde (2004, 2008), Perry et al. (2010). The current model incorporates an extended parameter space including velocity, radius, impact parameter, rotations, charge, density, magnetization and dipole interactions, among others.

The model was also modified to allow for ellipsoidal shapes by specifying three different axes for each monomer and then tracking their orientations within an aggregate and in space. The spheres and ellipsoids used in this study were of equal volume and mass. The ellipsoids were assumed to be convex prolate with an axis ratio of $3: 1: 1$. The particles modeled were silicates (of density $\rho=2.52 \frac{\mathrm{kg}}{\mathrm{m}^{3}}$ ) with radii of $R=2.88 \mu \mathrm{m}$ for spherical monomers and $R=6 \mu \mathrm{m}$ for the semi-major axis of the ellipsoidal monomers.

To create the aggregates, the model initially assumes a single monomer located at the origin of the coordinate system. A new monomer is randomly placed approximately $20 R$ from the initial particle, where $R$ is the maximum radius of a monomer or an aggregate measured from the COM. If the monomer is an ellipsoid, it is also given a random initial orientation. Velocities are established according to the thermal velocity

$v=\sqrt{\frac{k_{\mathrm{b}} T}{m}}$

where $T$ is the temperature of the gas in which the particles are immersed (set to $100 \mathrm{~K}$ for illustrative purposes), and $m$ is the mass of the particle. Particle trajectories are directed towards the COM of the initial particle with an offset ranging from 0 to $\left(R_{1}+R_{2}\right)$, the sum of the maximum radii of the two particles. As the incoming particle approaches within a distance $\left(R_{1}+R_{2}\right)$, the two closest monomers in each particle are examined to determine when overlap occurs. Conservation of angular momentum for the collision requires that off-center collisions lead to rotations of the resultant aggregate. Once overlap has occurred, the particles are combined to form a single new particle. Impacts which occur with larger offsets will induce faster rotations in this model. In the low-velocity regime examined, particles are assumed to stick at the point of contact and be held together via van der Waals forces, i.e. the so called hit-and-stick regime.
Aggregates are grown using both the CCA and PCA collision mechanisms. For each regime, two populations were assembled, one using spherical monomers and one using ellipsoidal monomers. For both aggregation mechanisms, smaller aggregates $(N \leq 20)$, were grown using PCA collisions. After each collision, particles are saved to a library and used as incoming aggregates for subsequent CCA collisions. For the PCA regime, aggregates were grown only through coagulation of single monomers with the target cluster. Aggregates were grown to a size $N \geq 1500$ across all populations.

Comparison of aggregates grown from spheres or ellipsoids are made through examination of the porosity, fractal dimension, compactness factor and friction time. Each of these is described below.

\subsection{Porosity}

The porosity is a measure of how much open space is contained in an aggregate and is defined as

$p=1-\frac{V}{V_{\mathrm{T}}}$

where $V$ is the total volume of the constituent monomers and $V_{\mathrm{T}}$ is the volume of a sphere with radius equal to the radius of gyration. This equation can be reduced, for monodisperse populations, to

$p=1-N\left(\frac{r}{R_{\mathrm{c}}}\right)^{3}$

where $r$ is the radius of a spherical monomer or the geometric mean radius of an ellipsoidal monomer (which are equal in this study), $N$ is the number of particles in the aggregate, and $R_{\mathrm{c}}$ is the characteristic radius of the aggregate found using the radius of gyration by $R_{\mathrm{c}}=\sqrt{\frac{5}{3}} R_{\mathrm{g}}$.

\subsection{Fractal dimension}

The mass fractal dimension, $F_{\mathrm{d}}$, is a measure of how completely a structure fills a three dimensional spatial region. The mass fractal dimension ranges from $\sim 1$ to $\sim 3$ which characterize open, linear structures and compact, spherical structures respectively. The mass fractal dimension is well described by a power-law relationship with respect to the aggregate radius

$m(r)=k r^{F_{\mathrm{d}}}$

where $m(r)$ is the mass of the aggregate contained within a radius $r$ and $k$ is a structural coefficient. The fractal dimension is calculated by plotting $m(r)$ versus $r$ for a given aggregate on a $\log -\log$ plot and determining the slope of the best-fit line.

\subsection{Compactness factor}

The compactness factor is an indication of the structural fluffiness or openness of an aggregate. Fluffy aggregates will have a greater probability of collision due to their higher geometric cross section. Additionally, the structure of an aggregate provides a measure of how well coupled it is to the surrounding gas. Particles well-entrained in the gas exhibit lower relative velocities, which can retard coagulation rates.

The compactness factor is calculated using the ratio of the volume of the constituent monomers to the volume of a sphere 
a

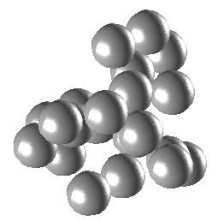

d

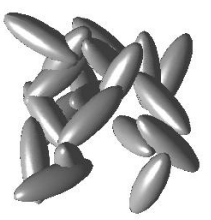

b

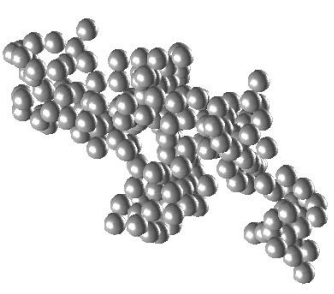

C

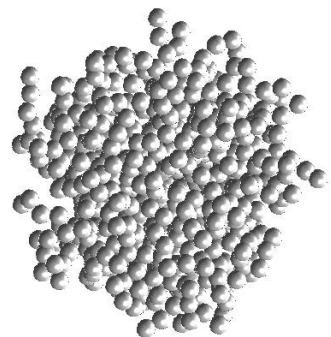

e
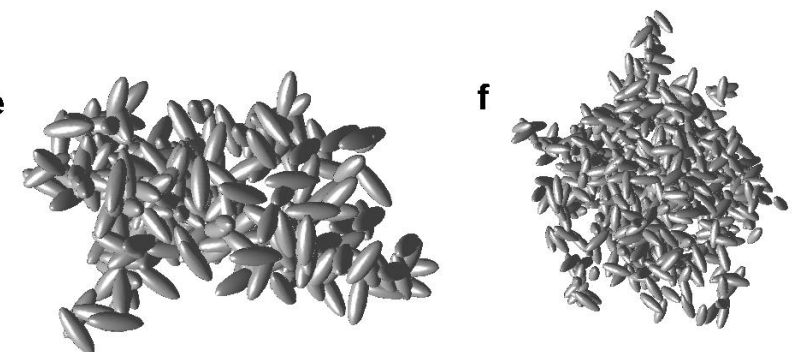

Fig. 1. Sample aggregates generated from the model described in the text a) PCA aggregate built from spheres $(N=20)$; b) CCA aggregate built from spheres $(N \sim 200)$; c) PCA aggregate built from spheres $(N \sim 700)$; d) PCA aggregate built from ellipsoids $(N=20)$; e) CCA aggregate built from ellipsoids $(N \sim 200)$; f) PCA aggregate built from ellipsoids $(N \sim 700)$.

with radius equal to the average projected cross-section of the aggregate, $A_{\sigma}$. The projected area is averaged over numerous orientations to minimize variance. Thus, the compactness factor is given by

$\Phi_{\sigma}=\frac{\sum_{i} r_{i}^{3}}{R_{\sigma}^{3}}$

where $r_{i}$ is the radius of the $i$ th monomer and the sum runs over all monomers in the aggregate. $R_{\sigma}$ is defined as the radius of a circle with area equal to the average projected cross-section Paszun \& Dominik (2009). For an ellipsoid, $r_{i}^{3}=a b c$, where $a$, $b$ and $c$ are the semi-axes of the monomer. High compactness factors are indicative of a more compact structure, while smaller compactness factors represent more open, fluffy structures.

\subsection{Friction time}

Aggregate growth is driven by relative velocities between particles. While for the smallest grains, Brownian motion is the dominant process leading to relative velocities, turbulent gas motion also can induce relative motion between aggregates Dominik et al. (2007b), and references within. The structure of the aggregate is a major factor in determining dust-gas interactions and overall timescale for growth processes.

The friction time, which depends upon the geometric crosssection, describes the aerodynamic properties of the dust particles and the strength of the interactions between the dust and the gas Kempf et al. (1999). The friction time, therefore, represents the time it takes for a particle to dissipate its velocity relative to the gas.

Particles with short friction times are well coupled to the gas and thus responsive to small eddies in the environment, usually resulting in low relative velocities between grains. The friction time is defined by

$\tau_{\mathrm{f}}=\frac{m}{\sigma} \frac{1}{\rho_{\mathrm{g}} v_{\mathrm{th}}}=\frac{N}{\sigma} \frac{m_{0}}{\rho_{\mathrm{g}} v_{\mathrm{th}}}$ where $\sigma$ is the cross-sectional area of the aggregate, $m_{0}$ is the monomer mass, $N$ is the number of monomers in an aggregate, $\rho_{\mathrm{g}}$ is the density of the gas and $v_{\text {th }}$ is the thermal sound speed Armitage (2010). Aggregates in a given astrophysical environment will be subject to the same environmental parameters, thus the differences in friction times depends on the ratio of the number of monomers to the cross-sectional area. Using $R_{\sigma}$ to calculate the cross-sectional area, the friction time is proportional to $\frac{N}{R_{\sigma}^{2}} \propto \Phi_{\sigma} R_{\sigma}$.

\section{Results}

Sample aggregates grown using the PCA and CCA mechanisms described in Sect. 2 are shown in Fig. 1. The differences in the morphology of the resulting aggregates and between populations are discussed below. Data sets for the following physical quantities have been binned according to size $(N)$ and averaged for clarity in examining trends. Due to the differing trends between aggregation regimes, CCA and PCA results shall be discussed separately.

In Figs. 2a,b, lines indicate that results from collisions assuming no rotation or off-center collisions. This data replicates the results in Bertini et al. A difference in two populations is seen when rotations (caused by off-center collisions) are allowed as indicated by the data points with their associated error bars. When rotations are not allowed, including an impact parameter, $b$, does not change the previously reported results, since an off-center impact is equivalent to an impact directed toward the COM along a different approach vector during ballistic collisions. Thus, the difference in physical characteristics for aggregates consisting of two monomer types is due only to the induced rotation.

The aggregate spin rates, shown in Fig. 3, indicate that the mechanical spin rate of the aggregates evolves similarly for both shapes, as would be expected for aggregates grown through impacts with particles of equivalent mass and speed. The magnitudes of the induced rotations differ by $\sim 10 \%$ for PCA collisions and $\sim 5 \%$ for CCA collisions. These differences are not large enough to produce the additional structural variations seen 

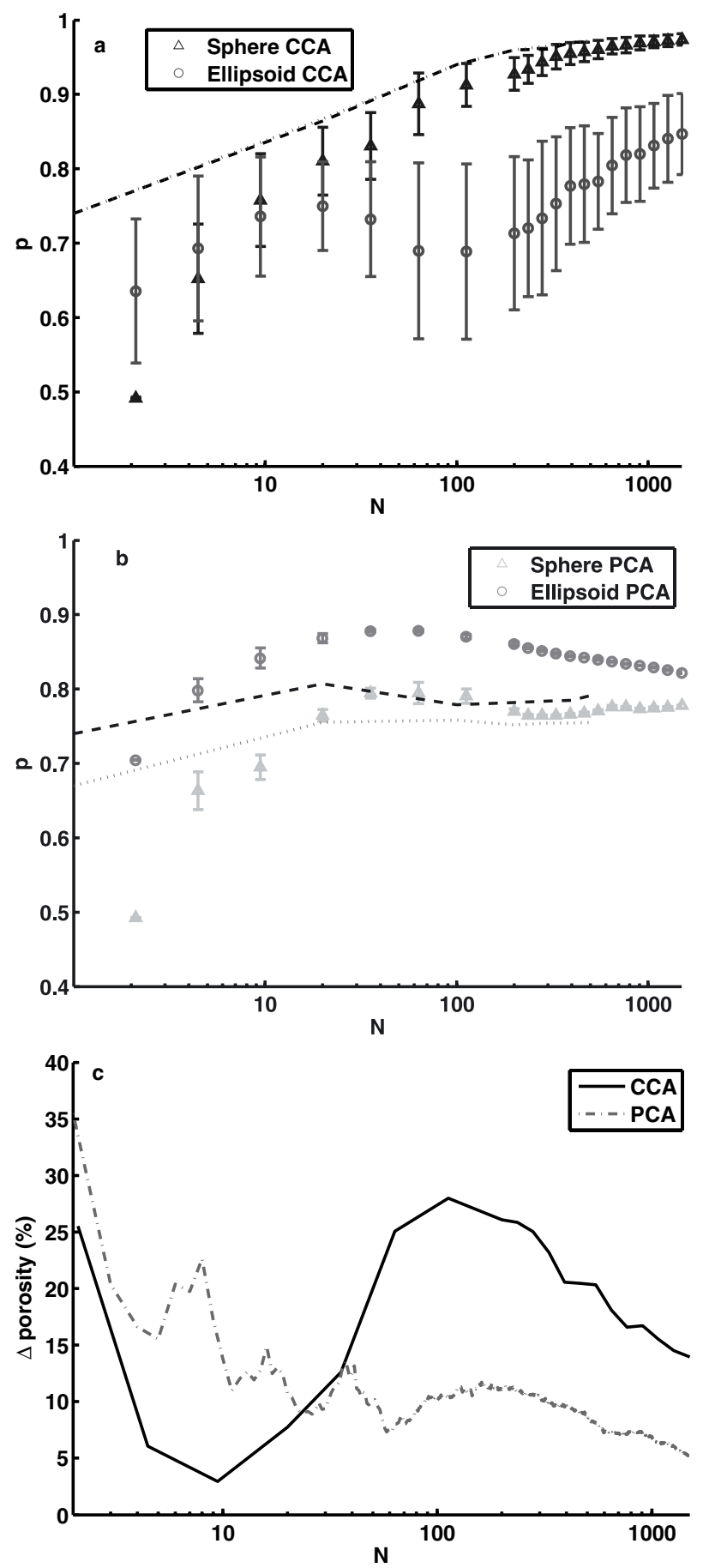

Fig. 2. Average porosities for a) populations built from CCA collisions, and b) populations built from PCA collisions. c) Percent difference between aggregates built using different monomer shapes within the same collision regime. The lines in a) and b) represent the porosities of populations grown without using an offset or rotation. The darker line corresponds to aggregates comprised of ellipsoidal monomers, while the lighter is for aggregates comprised of spherical monomers. In a) the differences between spheres and ellipsoids are so small that the trend lines coincide at all points.

in Figs. 2 and 4-6. This is again in agreement with Paszun \& Dominik (2006), within assumed limitations, who also found that rotations play a significant role in determining the structure of aggregates. In their work, Brownian rotations led to the formation of elongated aggregates, especially in the non-ballistic limit of dense gaseous environments.

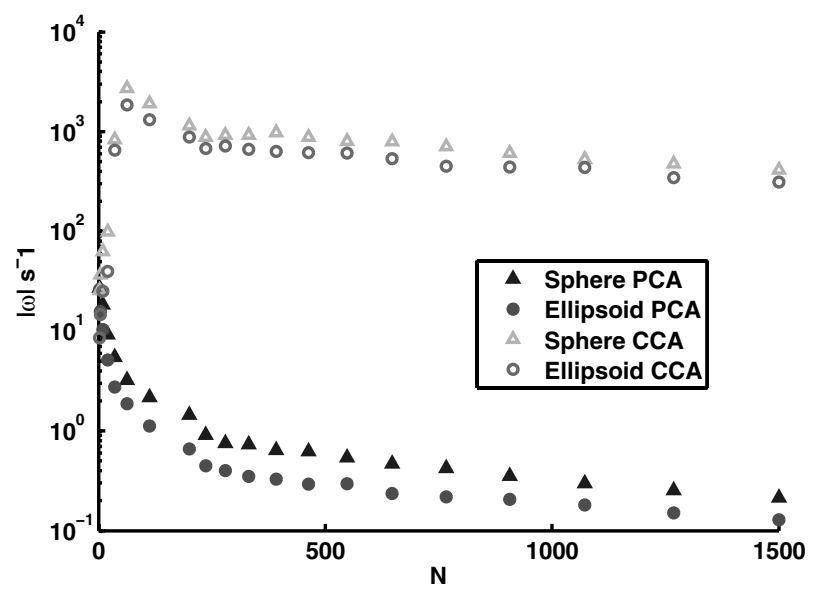

Fig. 3. Magnitude of rotations induced by off-center collisions during the aggregation process. Collisions between clusters are seen to produce much higher spin rates than collisions of a single particle with a cluster, as expected.
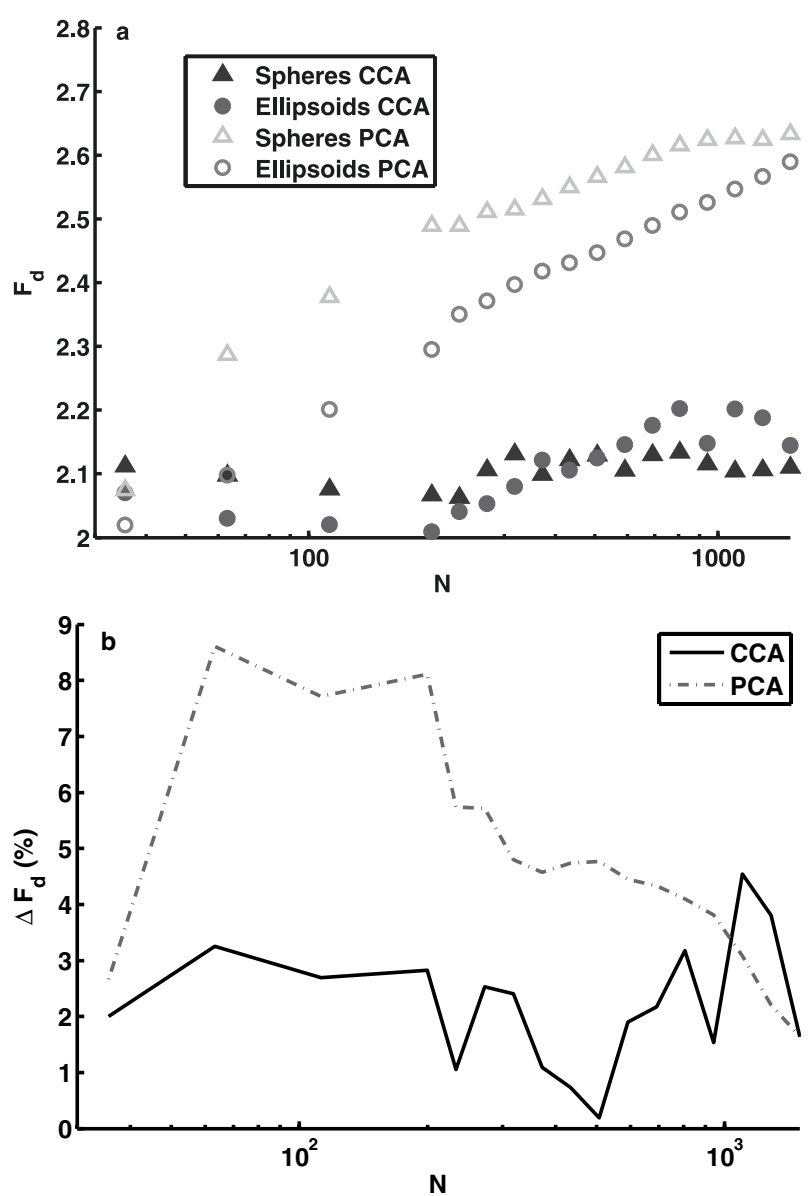

Fig. 4. Fractal dimensions of aggregates for a) CCA and PCA populations with b) percent differences between aggregates using the same growth regime.

\subsection{Cluster-cluster aggregation}

As shown in Fig. 2a, aggregates built from ellipsoids tend to be less porous than those built from spheres for CCA collisions, and have a greater variation for a given size. The average difference in porosity for CCA populations tends to almost $15 \%$. For comparison purposes, the dashed lines in Fig. 2a indicate the porosity of aggregates grown without offset or rotation. For 
J. D. Perry et al.: The influence of monomer shape on aggregate morphologies
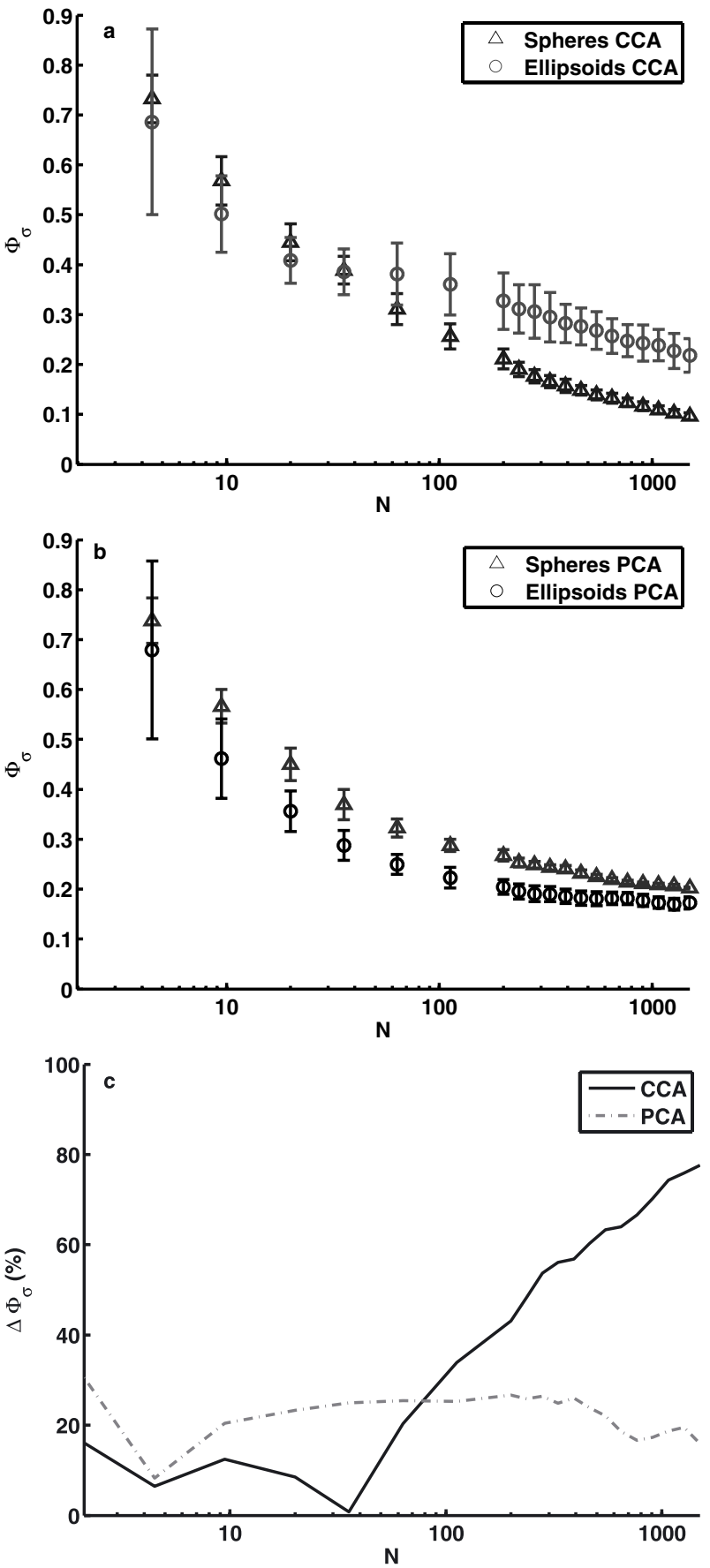

Fig. 5. Average compactness factor (with associated errors) for a) populations built using CCA collisions, and b) populations built using PCA collisions. c) Percent difference between aggregates built using different monomer shapes in the same collision regime.

this case, the results for ellipsoids and spheres overlap almost exactly, in agreement with the results reported in Bertini et al. (2009), where the average percent difference for the two populations was near zero.

Aggregates built from both monomer types exhibit fractal dimensions of approximately $D_{\mathrm{f}} \sim 2$ (Fig. 4a), in good agreement with the results for ballistic collisions as seen by Dominik $\&$ Tielens (1997). The difference between populations for this regime remains relatively low $(\sim 3 \%)$ at all sizes (Fig. $4 b$ ). Any small difference seen is likely due to the fact that fractal
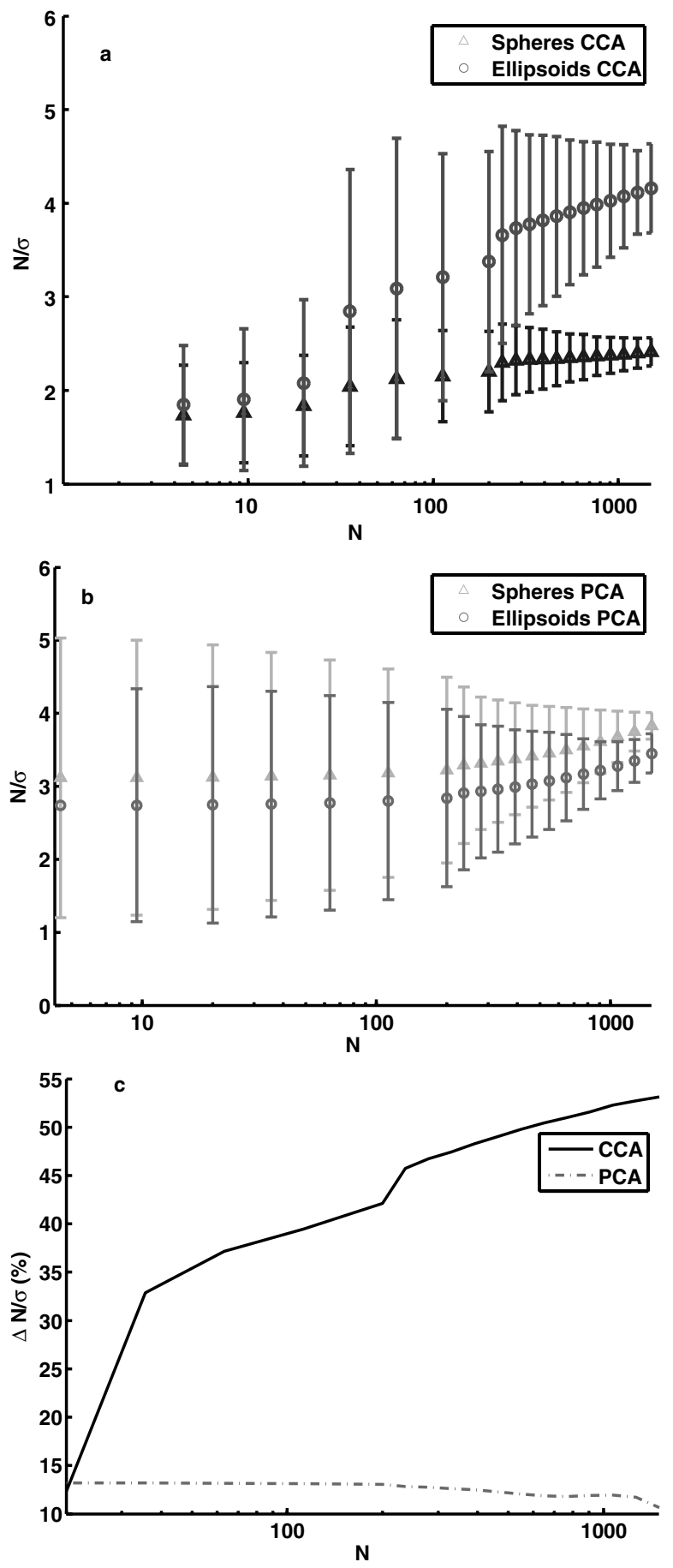

Fig. 6. Average friction times (with associated errors) calculated using a normalized geometric cross-section and for arbitrary environmental parameters for a) populations built using CCA collisions, and b) populations built using PCA collisions. c) Percent difference between aggregates built using different monomer shapes in the same collision regime.

dimension is not a good parameter for quantifying differences in the structure of these aggregates.

The compactness factor, as calculated by Eq. (5) and shown in Fig. 5a, indicates that aggregates comprised of spheres are much less compact than those grown using ellipsoids, in agreement with the trend for porosity. While the difference is relatively minor for small aggregates, $N \leq 20$, the difference becomes greater as aggregates grow larger (Fig. 5c). Additionally, the standard deviation is larger for ellipsoids than 
for spheres, implying a broader variation in structure for those aggregates.

The evolution of friction times, for the shapes used in this study, differ substantially as aggregates grow to larger sizes (Fig. 6a). The friction times for aggregates built from spheres increase only slightly with size, while those comprised of ellipsoids have significantly larger friction times for aggregates with $N>20$. This leads to a significant difference between aggregates formed from spheres and ellipsoids for all but the smallest aggregates (Fig. 6c), ellipsoidal aggregates more than doubling their response time as they grow. Coupled with the larger deviation seen in friction times across all sizes, this implies that turbulence could be a very effective mechanism for producing relative velocities which lead to coagulation for ellipsoidal particles.

\subsection{Particle-cluster aggregation}

In contrast to the CCA regime, PCA aggregation of ellipsoids generates aggregates that are more porous than aggregates consisting of spheres (Fig. 2b). In this case, the variance for a given size is quite small for both monomer shapes. The overall differences in the porosity of the two populations is $\sim 9 \%$, decreasing for larger aggregates. The dashed lines in Fig. $2 \mathrm{~b}$ again represent the resulting porosity when rotations induced by offset collisions are not allowed. In this case, the difference for the two populations is about $4 \%$ (Fig. 2c), in agreement with Bertini et al. (2009), who found a difference of $\sim 5 \%$.

Aggregates built under this regime exhibited higher fractal dimensions, as seen in Fig. 4a, verifying that aggregates which grow from single particle collisions are overall more dense than aggregates grown from collisions between clusters. The overall difference between the two populations remains relatively small, particularly when compared to the differences of the other quantities examined, and decreases as aggregates grow to larger size (Fig. 4c).

The compactness factors for PCA aggregates tend to a similar limiting value for large $N$ (Fig. 5b). This leads to a smaller, but not insignificant, difference between the compactness of these structures as exhibited in Fig. 5c. While the variance within both populations is comparatively small, aggregates built with ellipsoids still exhibit a larger range of compactness factors for a given size.

In contrast to the CCA regime, $\frac{N}{\sigma}$, and thus the friction times, of PCA aggregates evolve almost identically during growth (Fig. 6b). Populations also exhibit similar variance at all sizes. This leads to a difference between the populations which, while being more modest, is not negligible, averaging $\sim 12 \%$ for all sizes (Fig. 6c).

\section{Conclusions}

Populations of aggregates consisting of either spherical or ellipsoidal monomers grown using both PCA and CCA methods were compared according to porosity, fractal dimension, compactness factor and friction time. Two different monomer shapes, spheres and prolate ellipsoids with an axis ratio of $3: 1: 1$, were modeled using two aggregation mechanisms, PCA and CCA, producing aggregates that ranged from compact to fluffy, respectively.

Inclusion of an impact parameter and rotational degrees of freedom, neglected in a previous study, created aggregates with large structural differences. Differences between two populations is always smaller for PCA, ranging between 10-20\% for structural characteristics such as porosity, compactness factor, and $\frac{N}{\sigma}$, which determines friction time (Figs. 2, 5, 6). However, for CCA aggregation the differences exhibited in the compactness factor (Fig. 5) and friction times (Fig. 6) are significant as aggregates grow to larger size, and can have a large impact on the evolution of a protoplanetary disk or a molecular dust cloud.

Aggregates built using the PCA regime tend to be more compact than those grown using the CCA regime, as expected. However, differing trends are seen for the spheres and ellipsoids for the two aggregation regimes. In PCA aggregation, aggregates with ellipsoidal monomers are more porous, less compact, and have a smaller ratio of mass to cross-sectional area (indicating smaller friction times) than aggregates consisting of spheres. For CCA aggregation, just the opposite is true. However, for both cases, the aggregates with ellipsoidal monomers showed greater variation in the structural characteristics for a given size, which is due to the variation of the orientation of ellipsoids within aggregates.

These results illustrate the importance of considering the rotation of particles in astrophysical systems. Rotations may be caused by the conservation of angular momentum, as used in this model, or from Brownian rotation, which may play a significant role in dense gaseous environments Paszun \& Dominik (2006). In this study the difference in rotation rates between the two populations was small (Fig. 3), indicating that the magnitude of the rotation rates is probably not the cause of the large differences seen in the structural characteristics, especially for the CCA regime. While the difference in rotation rates for the PCA regime was comparable to the differences seen for structural characteristics $(\sim 10 \%)$, the effect was opposite of what is expected: aggregates consisting of spheres had higher spin rates, but were less porous and more compact than aggregates consisting of ellipsoids. Thus the shape of the monomers, coupled with the rotations of the aggregates, lead to the structural differences observed.

Evolution of populations of dust grains, such as in a protoplanetary disk, are usually studied using a statistical approach, where the detailed microphysics of collisions are not included. The Smoluchowski equation is a commonly used method to determine the evolution of the size distribution Ossenkopf (1993). The collision micro physics are encapsulated in the coagulation kernel given by

$Q\left(y_{i}, y_{i}^{\prime}\right)=A_{\text {coll }}\left(y_{i}, y_{i}^{\prime}\right) v_{\text {rel }}\left(y_{i}, y_{i}^{\prime}\right) S\left(y_{i}, y_{i}^{\prime}\right)$

where $A_{\text {coll }}$ denotes the collision cross section, $v_{\text {rel }}$ is the relative velocity between particles, $S$ is the sticking efficiency, and these functions depend on the independent aggregate parameters $y_{i}$.

The collision cross-section and the relative velocity between grains will be significantly different for aggregates consisting of either of the two monomer shapes, as indicated by the compactness factor (Fig. 5), and friction time (Fig. 6). Using the CCA mechanism, aggregates built from ellipsoids are more compact, indicating a smaller collision cross-section, which will tend to reduce coagulation rates. At the same time, large aggregates consisting of ellipsoids have greater friction times, indicating that these aggregates will be less well coupled to the gas. The large variations in friction times for a given size as well as the increasingly different friction times for grains of different sizes will increase relative velocities between grains, offsetting the former effect.

Greater relative velocities between grains also lead to higher impact energies, which are important to consider when including restructuring and fragmentation of aggregates during collisions Blum \& Wurm (2008). High energy collsions can lead to compactification of an aggregate, making it denser and less porous, 
whereas collisions with even higher energies can lead to local or global fragmentation of an aggregate. It is possible that these more compact structures would be less likely to undergo restructuring during high energy collisions.

\section{Future work}

Monodisperse ellipsoidal monomers lead to a broad range in aggregate morphology for a given size, with the variations for porosity, friction time and compactness factor being as large or larger than those seen for polydisperse spherical monomers Kempf et al. (1999). Realistically, individual particles in astrophysical environments will have a distribution of sizes. Further work will examine the structural differences of aggregates grown using polydisperse populations for various monomer shapes.

This study focused on a single alternative to the commonly used spherical monomers: prolate ellipsoids. Oblate ellipsoids and prolate ellipsoids with varying axis ratios within monodisperse and polydisperse size distributions should be modeled to gain a complete picture of the influence of monomer shape on ballistic collisions.

Finally, in many astrophysical environments, the immersion of dust grains in a plasma or radiative environment can lead to them becoming charged. To this end it is also useful to model the coagulation of non-spherical charged particles, which requires estimating the charge arrangement on the aggregate surface and the electrostatic interactions between grains. Charged aggregates require greater relative velocities to overcome the Coulomb barrier. Turbulence-driven coagulation of charged particles leads to collisions preferentially occurring between aggreagtes with large size differences Matthews et al. (2012). The large differences in friction times for aggregates with ellipsoidal monomers may lead to markedly different coagulation behavior. In addition to modeling coagulation in protoplanetary disks, charged grains may be employed to model mesospheric ice particles, which play a role in the formation of noctilucent clouds and are known to have a wide range of shapes, such as spheres, cylinders and disks Mendis et al. (2005).

\section{References}

Armitage, P. J. 2010, Astrophysics of Planet Formation (Cambridge University Press)

Bertini, I., Gutierrez, P. J., \& Sabolo, W. 2009, A\&A, 504, 625

Blum, J., \& Wurm, G. 2008, ARA\&A, 46, 21

Blum, J., Wurm, G., \& Kempf, S. 2000, Phys. Rev. Lett., 85, 2426

Dominik, C., \& Nubold, H. 2002, Icarus, 157, 173

Dominik, C., \& Tielens, A. G. G. M. 1997, ApJ, 480, 647

Dominik, C., Blum, J., Cuzzi, J. N., \& Wurm, G. 2007a, Protostars and Planets $\mathrm{V}, 783$

Dominik, C., Blum, J., Cuzzi, J. N., \& Wurm, G. 2007b, Growth of Dust as the Initial Step Toward Planet Formation

Hage, H. I., \& Greenberg, J. M. 1990, ApJ, 361, 251

Kempf, S., Pfalzner, S., \& Henning, T. K. 1999, Icarus, 141, 388

Matthews, L. S., \& Hyde, T. W. 2004, IEEE Trans. Plasma Sci., 32, 586

Matthews, L. S., \& Hyde, T. W. 2008, IEEE Trans. Plasma Sci., 36, 310

Matthews, L., Land, V., \& Hyde, T. W. 2012, ApJ, 744, 8

Mendis, D. A., Rosenberg, M., Wang, W.-H., \& Sorasio, G. 2005, J. Atmosph. Sol.-Terrest. Phys., 67, 1178

Ormel, C. W., Spaans, M., \& Tielens, A. G. G. M. 2006, A\&A, 461, 215

Ossenkopf, V. 1993, A\&A, 280, 617

Paszun, D., \& Dominik, C. 2006, Icarus, 182, 274

Paszun, D., \& Dominik, C. 2009, A\&A, 507, 1023

Perry, J. D., Matthews, L. S., \& Hyde, T. W. 2010, IEEE Trans. Plasma Sci., 38, 792

Skorov, Y. V., Keller, H. U., \& Rodin, A. V. 2008, Planet. Space Sci., 56, 660 